empirical data on ecological safety issues.

EPA officials, however, show no signs of moving in that direction. And USDA officials have recently moved toward simpler, instead of more stringent, regulatory procedures for evaluating proposals for

field testing transgenic plants. USDA officials, in fact, argue that a streamlined regulatory approach will "cut costs, encourage biotechnology innovations, and focus agency regulatory resources on the areas of greatest complexity." -Jeffrey L. Fox

\title{
No winners against AIDS
}

No therapy has proveditself against HIV, not a preventive vaccine nor a therapeutic vaccine nor any of the immunesystem-boosting treatments.
WASHINGTON, D.C.-Thequest to develop both preventive and therapeutic HIV vaccines is proving a frustrating enterprise. Equally frustrating are efforts to jump start the waning immune systems of HIVinfected individuals. Indeed, these AIDS-research frustrations were everywhere in evidence during the "First National Conference on $\mathrm{Hu}$ man Retroviruses and Related Infections" held here recently.

One theme that emerged at the conference, however, is that cytotoxic Tlymphocytes (CTLs) appear to play a prominent role in maintaining the relative health of most HIV-infected individuals who do not progress to full-blown AIDS. According to Thomas Harrer at Massachusetts General Hospital (Boston, MA), HIV-infected individuals who nonetheless have remained healthy for periods of 10 years or more show a strong CTL response against HIV. Their immune systems, moreover, tend to recognize a variety of specific HIV epitopes. Some of these epitopes, which are being evaluated, could prove "attractive targets for immunotherapeutic interventions," Harrer concludes.

Yet these evaluations will likely be complicated because the CTL response is not to whole viruses, or even viral proteins, but to small viral peptides. These viral peptides, furthermore, are presented to the immune system in conjunction with the body's complex and still poorly understood major histocompatibility complex (MHC) molecules. Indeed, variations in individual MHC molecules may help explain why some individuals are more susceptible to the ravages of HIV than others.

Another prominent conference theme is that none of the numerous HIV vaccines have distinguished themselves. Among firms clinically testing HIV-envelope-protein vaccines are Chiron (Emeryville, CA), Genentech (S. San Francisco, CA), and MicroGeneSys (Meriden, CT). Immune Response (San Diego, CA), for its part, is testing a killed-virus vaccine. According to Deborah Birx of the Walter Reed Army Institute of Research (Rockville, MD), "in our hands, there are no significant differences among the envelope-protein vaccines." She adds, though, that preliminary data from phase II trials of these vaccines indicate that some individuals are showing improved CTL functions and other signs of beneficial immune-system responses.

However, results from in vitro assays evaluating the envelope-protein vaccines "give us pause," notes Mary Lou Clements of the Johns Hopkins School of Hygiene and Public Health (Baltimore, MD). Even though the vaccines stimulate the production of neutralizing antibodies in recipient individuals and even though these antibodies react with laboratory strains of HIV, the antibodies react "poorly, if at all, with fresh field isolates of HIV," says Clements. "This may be the fault of the assays. But we have a lot of work to do to clarify these results."

Regarding the therapeutic use of HIV vaccines, Robert Schooley of the University of Colorado Health Sciences Center (Denver, CO) says that "we can say that they can be given safely, but efficacy is far from demonstrated. And we can't say that any one is more promising than the others."

In addition to these HIV vaccines, Ronald Desrosiers of Harvard Medical School's primate facility (Southboro, MA) is working on a live HIV vaccine that is genetically modified so that it cannot replicate. However, Desrosiers says that there are many obstacles to developing such a vaccine. And Sten Vermund of the National Institute for Allergy and Infectious Diseases (NIAID,
Bethesda, MD) agrees, stating that "there is no strong industry champion for a live, attenuated HIV vaccine." Yet the approach may be seen in a more favorable light if multideletion mutants prove as effective as the simpler single-deletion versions that have been tested so far, notes Vermund.

In fact, several live-virus vaccines based on viruses other than HIVincluding vaccinia and cowpoxare being studied. They are being engineered to carry genes specifying one or more HIV polypeptides. Yet none among this group has emerged as a front runner.

Another theme explored at the conference involves efforts by researchers to boost the immune response in HIV-infected individuals through the administration of cytokines. For example, when interleukin-2 was intermittently administered to a small group of these individuals, more than half showed a "striking" improvement in immune functions, including increases in CD4 T lymphocytes, according to Anthony Fauci, director of the NIAID. "I have to underscore that, although these are the most dramatic increases of CD4 cells of any intervention, we must be circumspect, because we have no clinical correlates," Fauci says.

Anothercytokineapproach involves effortstoexpand immune-systemcells ex vivo, before reinfusing them into patients. One such approach is described as "adoptive cellular immunity" by Judith Lieberman of the New England Medical Center (Boston, MA). Lieberman extracts an HIVinfected individual's T lymphocytes, treats them in vitro with cytokines and HIV-derived peptides to boost CTL activity, and then reinfuses them into patients. "We have had encouraging preliminary results, including no acute toxicity and significant enhancement of HIV-peptide-specific cytotoxicity," saysLieberman. "Some patients had sustained increases in CD4 cell counts for many weeks after a single infusion."

Nonetheless, despite these positive results, AIDS researchers inevitably come back to the conference's central theme. No therapy has emerged as a sure winner in the campaign against HIV, not a preventive vaccine nor a therapeutic vaccine nor any of the immune-system-boosting treatments. —Jeffrey L. Fox 